\title{
Enhanced Microstrip Guard Trace for Ringing Noise Suppression Using a Dielectric Superstrate
}

\author{
Yung-Shou Cheng, Student Member, IEEE, Wei-Da Guo, Student Member, IEEE, Chih-Pin Hung, \\ Ruey-Beei Wu, Senior Member, IEEE, and Daniel De Zutter, Fellow, IEEE
}

\begin{abstract}
Grounded guard traces are increasingly used to reduce the coupling-induced crosstalk, but the incurred ringing noise will strongly limit the performance for the microstrip structures. This paper describes the generation mechanism of the ringing noise and derives an analytical formula of the noise magnitude. Besides, an enhanced microstrip guard trace design is proposed to eliminate the ringing noise by covering the original microstrip structure with a superstrate of higher permittivity. A design space versus the superstrate thickness and the dielectric constant are constructed and in which, the guard trace needs be grounded at the two ends only without causing any ringing noise. Finally, the time-domain simulations and experiments are performed to verify the proposed concept.
\end{abstract}

Index Terms - crosstalk noise, ringing noise, guard trace, noise suppression, dielectric superstrate, signal integrity.

\section{INTRODUCTION}

$\mathrm{E}^{1}$ LECTROMAGNETIC coupling known as crosstalk nowadays has become more significant owing to the increasing speed and routing density of IC packages [1], [2]. Crosstalk noise is usually represented in terms of nearend crosstalk (NEXT) and far-end crosstalk (FEXT) [3]. Both are proportional to the mutual capacitive and inductive coupling, but in addition, FEXT is proportional to the length of the coupled interconnects (provided the lines do not become too long) and only exists in inhomogeneous environments, e.g. the microstrip structure [3].

The crosstalk noise can be reduced by increasing the spacing, decreasing dielectric thickness, using differential signaling, minimizing parallel run length, etc., but affecting the system cost as tradeoffs [3]. By increasing the capacitive coupling coefficients to equalize the even- and odd-mode velocities, the FEXT can be eliminated. Some approaches have been proposed, e.g., by employing the stub-alternated microstrip line [4] and depositing the extra dielectric layer over the microstrip substrate [5], [6], but fail to reduce the NEXT.

Another technique to reduce the crosstalk noise is the placement of guard trace, i.e., to shield the coupling between adjacent signal lines by inserting a grounded trace [7], [8]. Intuitively, it blocks a significant part of the mutual coupling, thus being capable of suppressing both the NEXT and FEXT. In a real layout, however, the guard trace is connected to the ground plane by only a few plated via barrels and the shielding performance is affected.

Considerable effort has been spent to evaluate the performance of guard traces [9]-[12]. These all reveal the phenomenon of multiple half-wavelength resonances between two adjacent shorting vias on the guard trace. As the main signal line is driven, these resonances result in severe ringing noise at both the near and far ends of the victim line. In order to analyze the effect of the guard trace, some methods based on the lumped-circuit tee structure [9] and the multi-mode model [13] have been presented. However, an analytical expression to quantify the maximum magnitude of ringing noise is still lacking.

Since the appearance of ringing noise may greatly degrade the system performance, one common remedy is to increase the number of shorting vias on the guard trace so that the first resonance can be shifted away from the frequency band of interest [10]-[12]. This actually implies a higher cost and may lead to restrictions on the circuit routing. Another way to prevent the ringing noise is making use of the so-called serpentine guard trace [14]. This kind of guard trace has a serpentine shape and requires to be terminated with matched loads at both ends. The vertical sections of the guard trace provide additional capacitive coupling between adjacent signal lines with little change in the inductive coupling. The FEXT can thus be suppressed without producing ringing noise. However, the NEXT becomes larger and some extra space is needed for fabrication, which seems not attractive in dense interconnects.

Furthermore, instead of using a large number of shorting vias, a new approach by inserting an overlying dielectric layer on top of the microstrip guard trace structure was proposed [15]. The overlying dielectric is intentionally inserted to equalize the phase velocities of different modes, which has been found to be efficient in eliminating the FEXT [5], [6]. It was found to be also effective in suppressing the ringing noise as well, with only two shorting vias placed at both ends [15].

By extending the idea in [15], this paper provides a more comprehensive investigation and presents a systematic design procedure. It is organized as follows. In Section II, the generation mechanism of the ringing noise is fully explained and then an analytic formula is derived based on a multimode analysis to accurately predict the noise magnitude. Section III focuses the discussion on the enhanced microstrip guard trace structure following which a design graph is constructed to reveal the guard trace design with the minimum number of vias. Comparisons between simulated and measured results are presented for verification of the design concept in Section IV, followed by brief conclusions in Section V.

\section{Modeling AND ANALYSis OF RINGING NoISE}

Fig.1 illustrates the schematic diagram of two parallel 


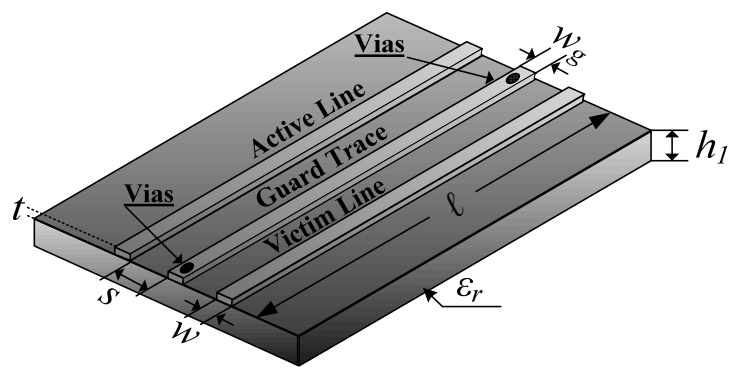

Fig. 1. Schematic diagram of two parallel microstrip lines separated by a via-grounded guard trace.

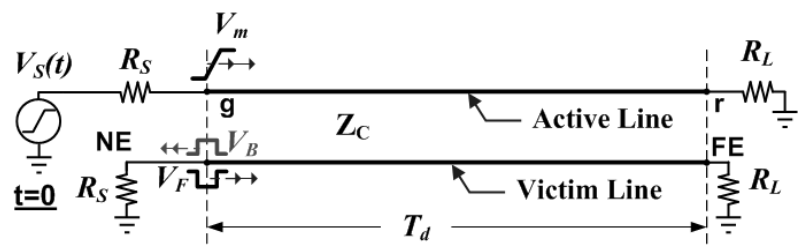

(a)

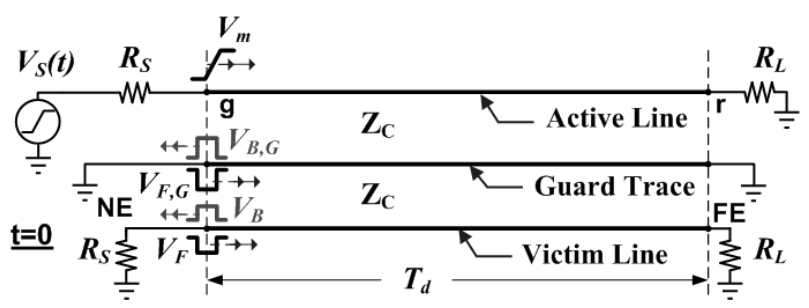

(b)

Fig. 2. Graphical representation of forward and backward propagating crosstalk noise in (a) coupled lines and (b) coupled lines with inserted guard trace.

microstrip lines separated by a guard trace which is grounded by two plated vias at both ends. It is clear that the shorting vias cause resonances at certain frequencies, leading to a series of ringing noise on the NEXT and FEXT waveforms [10]. This section investigates the generation mechanism both qualitatively and quantitatively.

\section{A. Qualitative Description}

Crosstalk is frequently encountered in bundles of long lines, backplane wirings, and circuit boards due to electromagnetic coupling between parallel transmission lines. A popular illustrative model based on wave tracing has been proposed to predict the crosstalk waveforms for two coupled transmission lines with matched termination [2].

Consider a ramped step pulse with amplitude $V_{m}$ and rise time $T_{r}$ propagating down the active line. The forward $\left(V_{F}\right)$ and backward $\left(V_{B}\right)$ propagating crosstalk noise will be induced on the victim line as shown in Fig. 2(a). $V_{B}$ propagates toward the near end of the victim line to form the NEXT. It is proportional to the sum of the capacitive and inductive coupling coefficients $k_{\mathrm{C}}$ and $k_{\mathrm{L}}$ defined below in (2a), with a total duration equal to the round trip time of the transmission line. $V_{F}$ propagates in the same direction as the main signal on the active line and appears

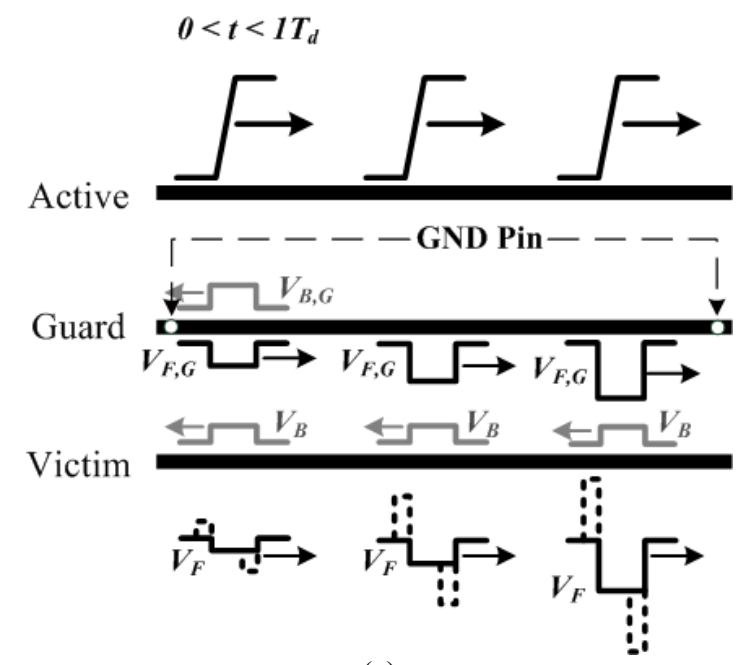

(a)

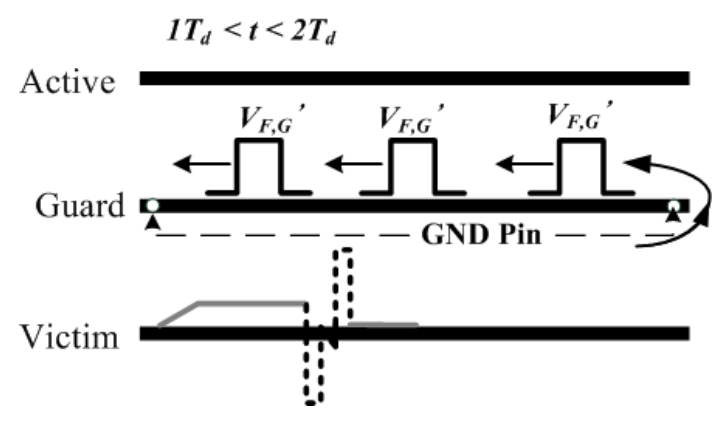

(b)

Fig. 3. Generation mechanism of ringing noise by forward propagating crosstalk on guard trace during (a) $0<t<T_{d}$ and (b) $T_{d}$

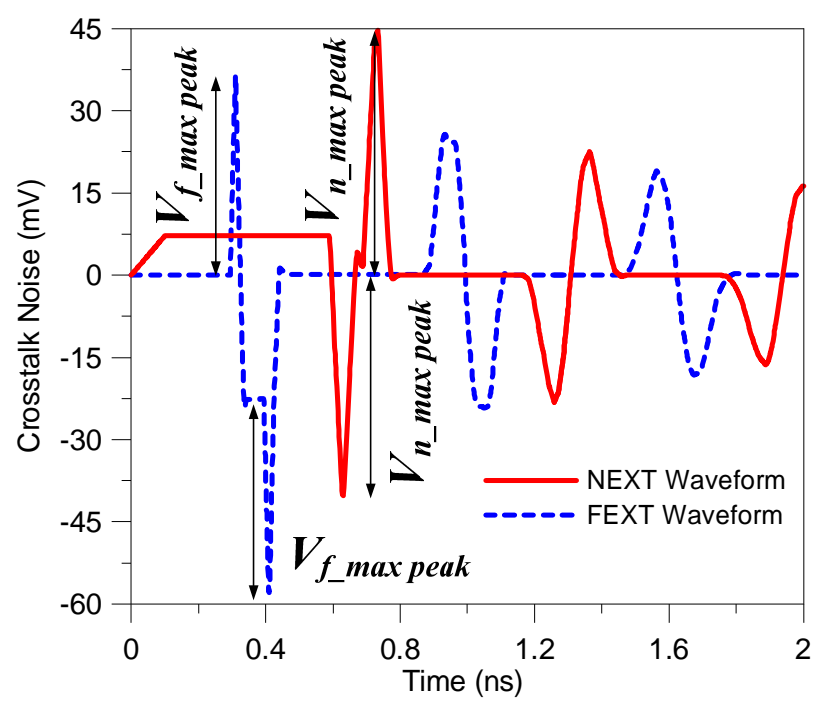

Fig. 4. Simulated ringing noise at near- and far-end crosstalk waveforms for a traditional microstrip guard trace with two ideal grounded vias at both ends.

at the far-end on the victim line as the FEXT. It is proportional to the difference between the capacitive and inductive coupling coefficients. Its waveform is proportional to the derivative of the pulse along the active line, with a total duration equal to the rise time. This description is valid provided the lines are weakly coupled, i.e., $k_{\mathrm{C}}$ and $k_{\mathrm{L}}$ must remain sufficiently small, but in general this assumption is met in the applications considered here. 


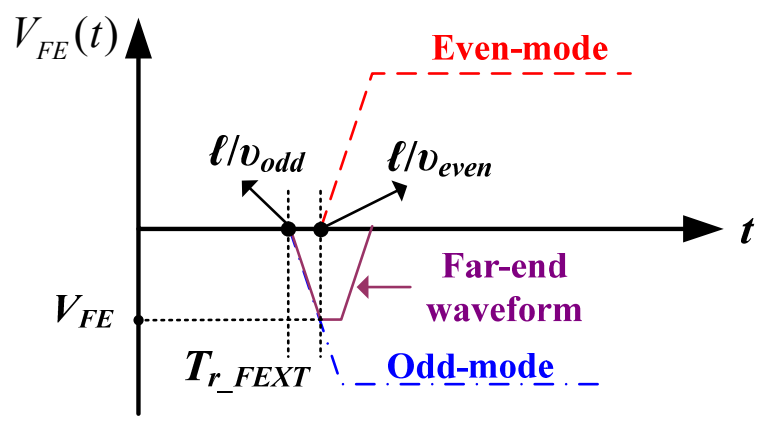

Fig. 5 Diagram of the far-end crosstalk resulting from the superposition of even and odd modes with unequal velocities after propagating over a distance $\lambda$.

For simplicity, the two shorting vias on the guard trace in Fig. 1 are regarded as ideal shorts with negligible inductive effect as shown in Fig. 2(b). The same idea described above is utilized once more to analyze the threeline transmission lines with the central line ideally grounded at the two ends only. As mentioned earlier, the forward and backward crosstalk noise will be induced on both victim line and guard trace simultaneously. To facilitate the description, $V_{F, G}$ and $V_{B, G}$ denote the forward and backward crosstalk noise traveling on the guard trace, respectively. When $V_{F, G}$ propagates toward the right end, it induces another forward crosstalk on the victim line. Note that the resultant pulse shape depends on the time derivative of $V_{F, G}$ and shows two spikes, one going up and the other going down as indicated by the dotted lines in Fig. 3(a)

Once $V_{F, G}$ reaches the shorting via at the right end, its voltage polarity is inverted and it propagates toward the left end. The reflected pulse, called $V_{F, G}$, also induces forward crosstalk on the victim line, but now appears at the near end. Following the same procedure, two spikes appear at the near end after twice the time delay $\left(2 T_{d}\right)$ as shown in Fig. 3(b). Consequently, there are a series of spikes at both the near and far ends.

To validate the qualitative analysis, numerical simulations are performed with the circuit model shown in Fig. 2(b). Consider a traditional microstrip guard trace structure depicted in Fig. 1 with $w=w_{g}=60 \mu \mathrm{m}, s=50 \mu \mathrm{m}$, $h_{l}=30 \mu \mathrm{m}, t=15 \mu \mathrm{m}, \varepsilon_{r}=3.4$, and line length $\ell=60 \mathrm{~mm}$. The substrate and conductors are assumed to be lossless. The input ramped step pulse has a $2 \mathrm{~V}$ amplitude and a $100 \mathrm{ps}$ rise time, while both the active and victim lines are terminated with matched impedances $R_{S}=R_{L}=50 \Omega$. The NEXT and FEXT waveforms simulated by the circuit simulator HSPICE [16] are shown in Fig. 4. It can be seen that a series of voltage spikes appear at both the near and far ends on the victim line as predicted by the qualitative analysis. It deserves mentioning that all these spikes come from the forward crosstalk mechanism.

\section{B. Quantitative Analysis}

For two coupled microstrip lines as shown in Fig. 2(a), the NEXT and FEXT can be approximated as [2].

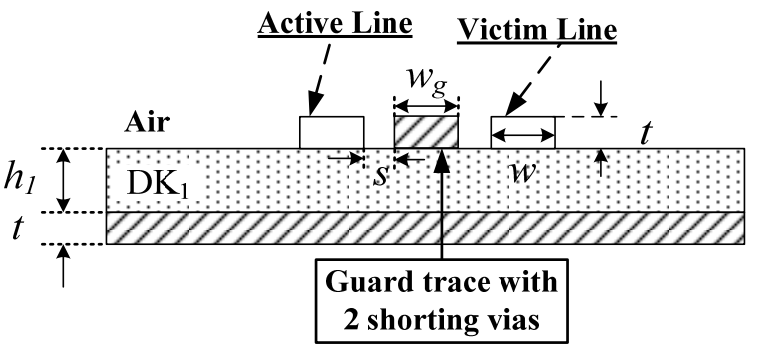

(a)

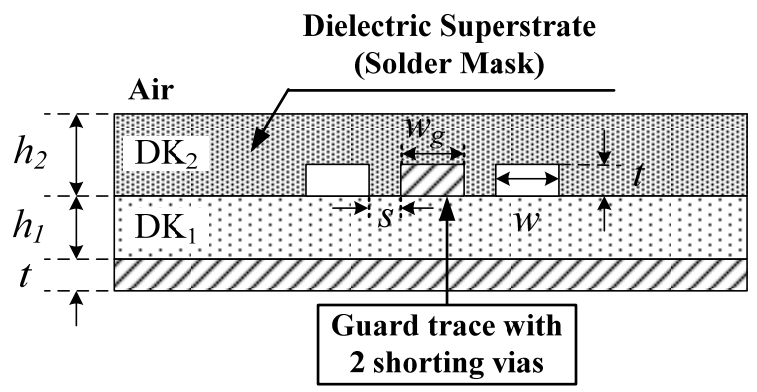

(b)

Fig. 6. Cross section of microstrip guard trace structure (a) without and (b) with dielectric superstrate.

$$
\begin{aligned}
& V_{N E}(t)=\frac{1}{4} \cdot K_{b} \cdot\left[V_{g}(t)-V_{g}\left(t-2 T_{d}\right)\right] \\
& V_{F E}(t)=\frac{-1}{2} \cdot K_{f} \cdot T_{d} \cdot \frac{d}{d t}\left[V_{g}\left(t-T_{d}\right)\right]
\end{aligned}
$$

where $V_{g}(t)$ is the voltage at the sending end ' $\mathrm{g}$ ' of the active line, $T_{d}$ is the time delay of the transmission line, and the backward and forward coupling coefficients are defined by

$$
\begin{aligned}
& K_{b}=k_{L}+k_{C}=\frac{L_{12}}{L_{22}}+\frac{\left|C_{12}\right|}{C_{22}} \\
& K_{f}=k_{L}-k_{C}=\frac{L_{12}}{L_{22}}-\frac{\left|C_{12}\right|}{C_{22}}
\end{aligned}
$$

Here, $k_{L}$ and $k_{C}$ denote the inductive and capacitive coupling coefficients, respectively. From (1b) it follows that the FEXT takes a rectangular pulse shape whose width equals the rise time of the source [17]. However, a detailed analysis reveals that it is trapezoid with finite rise/fall time [18].

To obtain the rise/fall time of the FEXT, assume that the two coupled transmission lines are identical. It is well known that the signal propagating along the coupled transmission lines can be decomposed into an even and an odd mode. Since the electric field of the even mode is more prominent in the substrate, its propagation speed is slower than that of the odd mode. It is this slight difference in the propagation speeds that causes the FEXT. Furthermore, the difference in the propagation delay between the even and odd modes

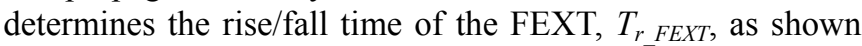
in Fig. 5, where $v_{\text {even }}$ and $v_{\text {odd }}$ are the velocities of the even and odd modes, respectively.

Since this delay difference is usually much smaller than $T_{r}$, it is not difficult to infer from Fig. 5 that the maximum FEXT satisfies 


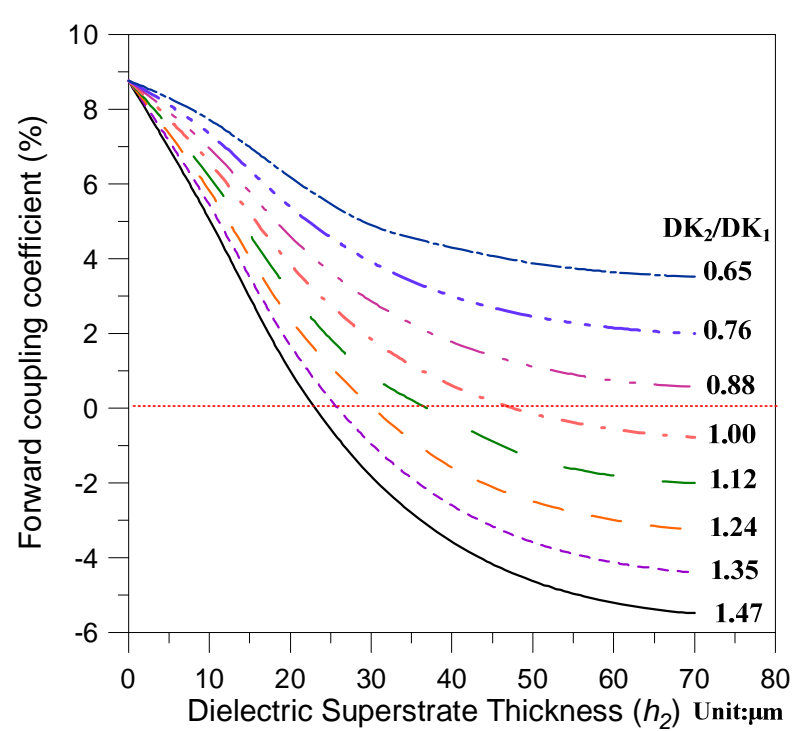

Fig. 7. Forward coupling coefficient $\left(K_{f, g}\right)$ versus superstrate thickness $\left(H_{2}\right)$ with the dielectric constant $\mathrm{DK}_{2}$ to $\mathrm{DK}_{1}$ ratio as a parameter.

$$
V_{F E}=-\frac{V_{m}}{2} \cdot \frac{T_{r_{-} F E X T}}{T_{r}}
$$

From (1b), this value is also given by

$$
V_{F E}=\frac{-1}{2} \cdot K_{f} \cdot T_{d} \cdot \frac{V_{m}}{T_{r}}
$$

Comparing (3) and (4) yields an approximation to the rise/fall time of the FEXT, i.e.,

$$
T_{r_{-} F E X T} \approx T_{d} \cdot\left|K_{f}\right|
$$

Following the qualitative analysis in part $\mathrm{A}$ of this section, the peaks of the ringing noise at both near and far ends are directly related to the time derivative of the forward crosstalk on the guard trace. The absolute value of the first voltage peak can be approximated by applying (4), but with the $V_{\mathrm{m}}$ and $T_{\mathrm{r}}$ in the rightmost term replaced by $V_{F E}$ in (4) and $T_{r_{-} F E X T}$ in (5), respectively. However, it should be mentioned that the difference in propagation delay is not necessarily smaller than the rise/fall time of the FEXT in (5). A correction factor $\kappa$ which is smaller than one should be introduced. As a result, the waveform is a triangular pulse and its maximum value is given by

$$
V_{n_{-} \max \text { peak }}=\kappa \cdot \frac{1}{4} \cdot\left|K_{f, g}\right| \cdot T_{d} \cdot \frac{V_{m}}{T_{r}}
$$

Here, $K_{\mathrm{f}, \mathrm{g}}$ is the forward coupling coefficient defined in (2b) with respect to the active line and the guard trace.

\section{Rigorous Three-Line Analysis}

An accurate determination of the correction factor $\kappa$ in (6) is impossible without resorting to a rigorous multimode analysis. Consider a system of three uniformly coupled microstrip lines shown in Fig. 2(b). The propagating signal can be decomposed into three fundamental modes: $\mathrm{EE}, \mathrm{OO}$ and $\mathrm{OE}$ modes [19]. The corresponding voltage transformation matrix is given by:

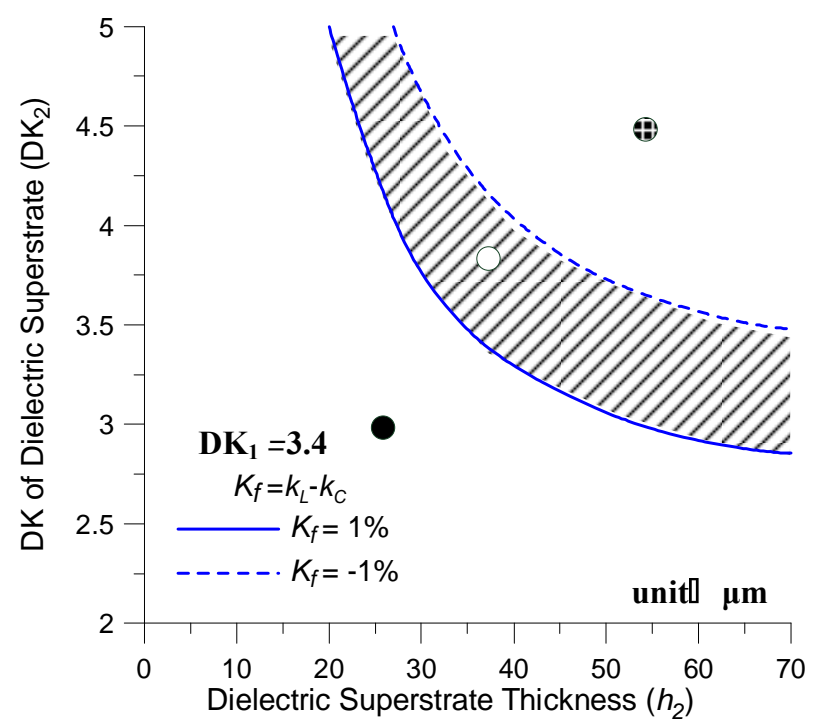

Fig. 8. Design solution space of minimum ringing noise for microstrip guard trace.

$$
T_{V}=\left[\begin{array}{ccc}
1 & 1 & 1 \\
\eta_{1} & \eta_{2} & 0 \\
1 & 1 & -1
\end{array}\right]
$$

Each column denotes the line voltages on the three lines for each mode. It corresponds to an eigenvector of the multiplication matrix of the inductance and capacitance matrices, i.e., $[L] \square[C]$. The value $\eta_{1}$ is positive and $\eta_{2}$ is negative, approximately satisfying [20]

$$
\eta_{1} \cdot \eta_{2}=-2
$$

Let $V_{\text {Line }}$ be a column vector formed by $V_{1}, V_{2}$, and $V_{3}$ which are the line voltages launched on the active line, guard trace, and victim line, respectively. It can be related to the column vector $V_{\text {Mode }}$ of the modal voltages $V_{\mathrm{EE}}, V_{\mathrm{OO}}$, and $V_{\mathrm{OE}}$ of the three modes by

$$
V_{\text {Line }}=T_{V} \cdot V_{\text {Mode }}
$$

Note that only EE and OO modes appear on the guard trace according to (8). In the multi-mode analysis, each mode propagates along the coupled lines independently and has its specific modal capacitance and inductance and thus modal impedance and propagation velocity, e.g., for $\mathrm{EE}$ and $\mathrm{OO}$ modes,

$$
\begin{aligned}
& v_{e e}=1 / \sqrt{\left(L_{22}+\frac{2}{\eta_{1}} L_{12}\right) \cdot\left(C_{22}+\frac{2}{\eta_{1}} C_{12}\right)} \\
& v_{o o}=1 / \sqrt{\left(L_{22}-\eta_{1} L_{12}\right) \cdot\left(C_{22}-\eta_{1} C_{12}\right)}
\end{aligned}
$$

Here, $L_{i j}$ and $C_{i j},(i, j=1,2.3)$ are elements of the $3 \times 3$ inductance and capacitance matrices of the three-line system.

The difference of the propagation delay between the EE and $\mathrm{OO}$ modes determines the rise/fall time of the FEXT on the guard trace. By dropping second-order small terms, the rise/fall time of the FEXT on the guard trace can be obtained 


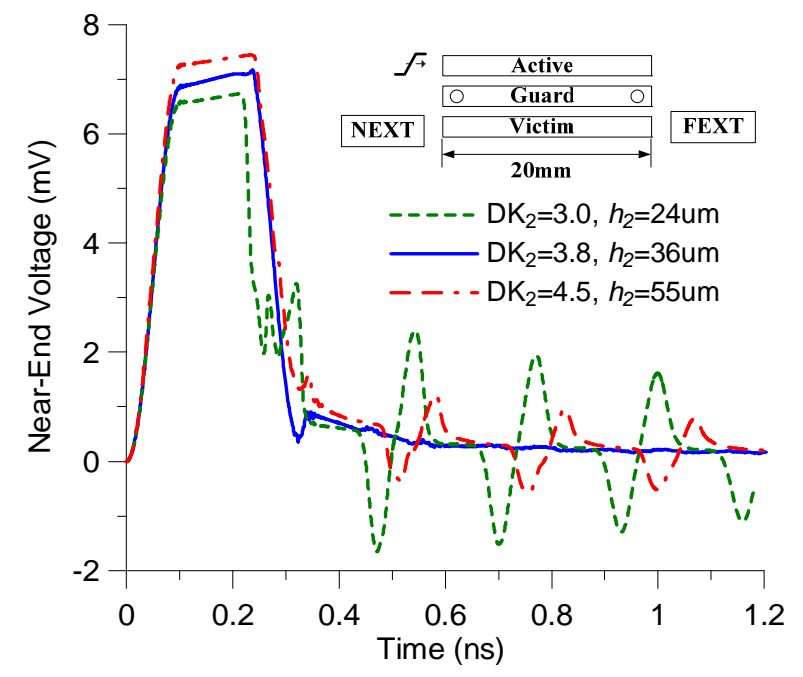

Fig. 9. Simulated near-end waveform for three different cases marked in Fig. 8.

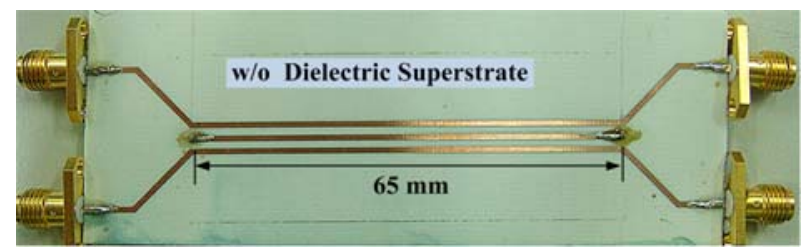

(a)

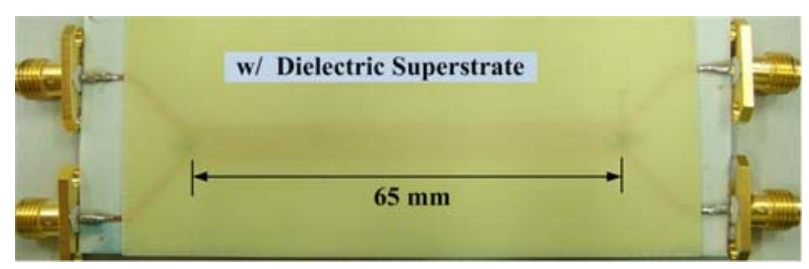

(b)

Fig. 10. Photographs of microstrip guard trace test vehicles (a) without and (b) with dielectric superstrate.

as

$$
T_{r_{-} F E X T}=\frac{\ell}{v_{e e}}-\frac{\ell}{v_{o o}} \approx \frac{\eta_{1}^{2}+2}{2 \eta_{1}} \cdot T_{d} \cdot\left(\frac{L_{12}}{L_{22}}-\frac{\left|C_{12}\right|}{C_{22}}\right)
$$

Consequently, the correction factor in (6) is given by

$$
\kappa=\frac{2 \eta_{1}}{\eta_{1}^{2}+2}
$$

Given the geometric dimensions of the three-line microstrip lines shown in Fig. 4, it is not difficult to calculate the capacitance and inductance matrices from the numerical simulation. Solving the eigensolution of $[L] \square[C]$ yields $\eta_{1}=1.22$ for the present example. The value of the first voltage peak magnitude at the near end waveform can be obtained using (6) and (12). Table I lists the predicted value and those found by a HSPICE simulation. The good agreement reveals that the amplitude of the ringing noise is proportional to the forward coupling coefficient $\left(K_{f, g}\right)$. The formula derived here for lossless lines also provides an upper bound of the ringing noise in lossy lines.

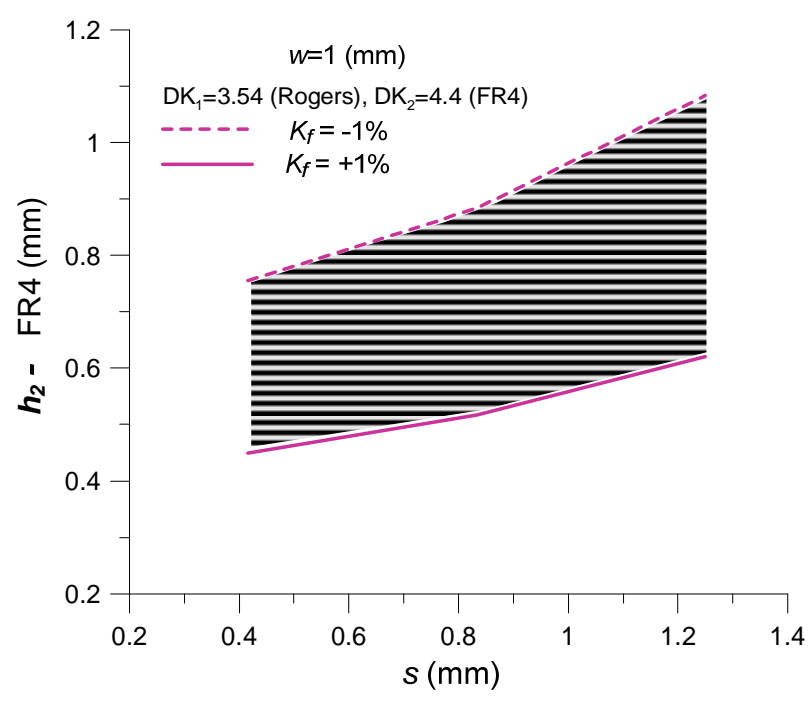

Fig. 11. Design solution space for a microstrip guard trace test structure.

Table I

Comparison of peak voltages at near-end waveform between approximation formula and HSPICE simulation

\begin{tabular}{|c|c|}
\hline & $V_{n_{\text {_max }} \text { peak }}$ \\
\hline Approximation & $49.75 \mathrm{mV}$ \\
\hline HSPICE Simulation & $\begin{array}{c}46 \mathrm{mV} \\
\text { (loss tangent }=0, \text { PEC) }\end{array}$ \\
\hline HSPICE Simulation & $\begin{array}{c}41 \mathrm{mV} \\
\text { (loss tangent }=0.02, \text { copper) }\end{array}$ \\
\hline
\end{tabular}

\section{Design SPACE FOR Minimum Ringing NoISE}

According to the aforementioned analysis, the ringing noise originates from the forward crosstalk mechanism on the guard trace, which is proportional to the difference between the inductive and capacitive coupling coefficients. For the general coupled microstrips shown in Fig. 6(a), the inductive coupling is always larger than the capacitive coupling. This results in significant ringing noise in such an inhomogeneous environment. However, a dielectric superstrate, called solder mask in the industry, is usually placed on top of the microstrip structure for manufacturing consideration as shown in Fig. 6(b). The difference between the inductive and capacitive coupling can be reduced by increasing the mutual capacitance but with fixed mutual inductance [5]. Therefore, the ringing noise can be eliminated even in an inhomogeneous environment when the forward coupling coefficient between active line and guard trace tends to zero.

Consider the original microstrip substrate covered by a superstrate having the cross section as shown in Fig. 6(b), where $s=50 \mu \mathrm{m}, w=w_{g}=60 \mu \mathrm{m}, h_{1}=30 \mathrm{um}, t=15 \mathrm{um}$. Figure 7 shows the calculated forward coupling coefficient $\left(K_{f, g}\right)$ versus the thickness of the dielectric superstrate $\left(h_{2}\right)$ with the dielectric constant ratio, $\mathrm{DK}_{2} / \mathrm{DK}_{1}$, as a parameter, where $\mathrm{DK}_{1}$ and $\mathrm{DK}_{2}$ denote the dielectric constants of substrate and superstrate, respectively. 


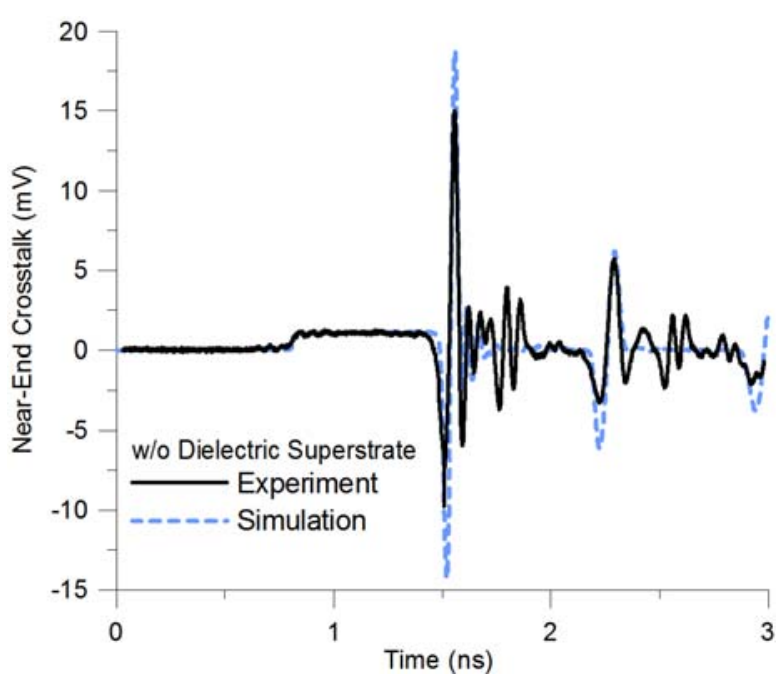

(a)

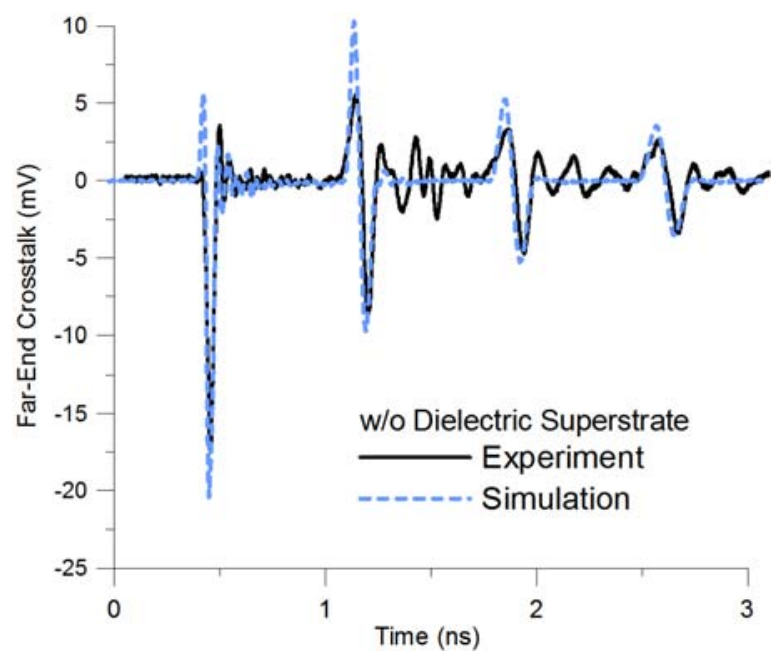

(b)

Fig. 12. Comparison between simulated and measured (a) NEXT and (b) FEXT waveforms of a microstrip guard trace test structure.

It is found that inductive and capacitive coupling coefficients are identical if the superstrate thickness and its dielectric constant $\left(\mathrm{DK}_{2}\right)$ are suitably chosen. A superstrate with higher dielectric constant and larger thickness usually corresponds to a higher capacitive coupling. If the dielectric constant of the superstrate is smaller than that of the substrate, i.e., $\mathrm{DK}_{2}<\mathrm{DK}_{1}$, the capacitive coupling is always smaller than the inductive coupling. As a result, this design is only possible when the dielectric constant of the superstrate is higher than that of the microstrip substrate.

For a fixed substrate with $\mathrm{DK}_{1}=3.4$, the design space for the superstrate to yield less than $\pm 1 \%$ difference between the capacitive and inductive coupling is illustrated by the shaded region in Fig. 8. In this region, the guard trace needs only to be grounded at both ends to obtain good performance with negligible ringing noise. It is a useful design chart for the PCB manufacturer.

To verify the accuracy of the above design solution space, three cases from Fig. 8 are selected to perform

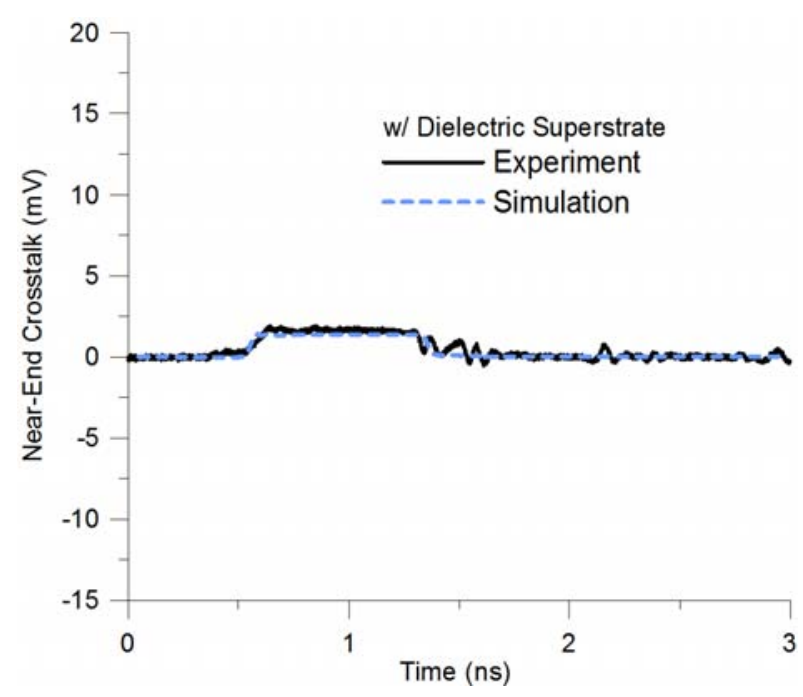

(a)

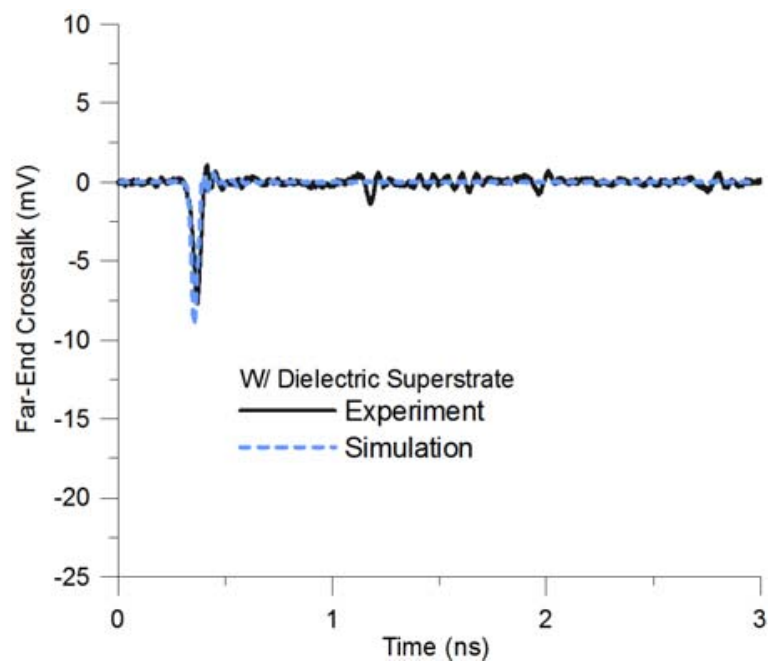

(b)

Fig. 13. Comparison between simulated and measured (a) NEXT and (b) FEXT waveforms of a microstrip guard trace with dielectric superstrate.

time-domain simulation using CST Microwave Studio [21], a $3 \mathrm{D}$ finite integration simulator. An input signal with 1V amplitude and 100ps rise time is applied to the active line as depicted in the upper right corner of Fig. 9. The near-end voltage waveforms for the three cases are shown in Fig. 9. It is validated that the dielectric superstrate designed within the solution space can successfully reduce the crosstalk without causing any ringing noise, although the guard trace is grounded at the two ends only.

\section{EXPERIMENTAL VERIFICATION}

To verify the proposed methodology for ringing noise suppression, as suggested in section III, the waveforms of the near- and far-end crosstalk on the victim line are measured and compared to the simulation results. Consider the microstrip guard trace structure in Fig. 10(a) having the cross section shown in Fig. 6(a). For ease of reach in our laboratory, the Rogers RO4003 with dielectric constant 3.54 and height $0.5 \mathrm{~mm}$ is chosen as the substrate while the FR4 with 
dielectric constant 4.4 is used as superstrate. The microstrips have width $1 \mathrm{~mm}$, thickness $0.035 \mathrm{~mm}$, and trace length $65 \mathrm{~mm}$. To equalize the capacitive and inductive coupling coefficients, the solution space versus the FR4 thickness $\left(h_{2}\right)$ and trace separation $(s)$ is constructed in Fig. 11. Since the FR4 board thickness available is $0.8 \mathrm{~mm}$, the separation is chosen as $1 \mathrm{~mm}$. The microstrip structure is also fabricated for experimental verification as shown in Fig. 10(b). The cross sectional view of the test vehicle is shown in Fig. 6(b).

The NEXT and FEXT waveforms on the victim line are measured by the time-domain reflectometer Tektronix CSA8000B. The launching voltage source is assumed to have a rise time of $35 \mathrm{ps}$ and a magnitude of $0.5 \mathrm{~V}$. Figures 12 and 13 show the comparison between the simulated and measured waveforms on the victim line at the near and far ends, respectively. Simulation and measurement are in good agreement.

In Fig. 12, it is observed that the undesired ringing noise spoils the efficiency of the crosstalk reduction by the guard trace. Although not shown here, the NEXT and FEXT are $1.8 \mathrm{mV}$ and $21.1 \mathrm{mV}$, respectively, without using the guard trace. If the guard trace is not suitably grounded or well designed as in the present case, the maximum NEXT and FEXT become as large as $16 \mathrm{mV}$ and $17.5 \mathrm{mV}$, respectively.

With a suitable superstrate design proposed in this paper, the guard trace can successfully reduce the crosstalk without causing ringing noise as evident in Fig. 13. The NEXT and FEXT have been reduced to $1.6 \mathrm{mV}$ and $7.6 \mathrm{mV}$, or $11.1 \%$ and $64 \%$ improvement, respectively, as compared with the original coupled microstrips. Although the present superstrate design equalizes the capacitive and inductive coupling coefficients between active line and guard trace, it is also worth noting that the two coupling coefficients between active and victim lines are not the same. Hence, there is still a nonzero far-end crosstalk. Nonetheless, its magnitude is significantly reduced as compared with that in the original coupled microstrips without the guard trace.

\section{CONCLUSIONS}

It is a common practice to include a grounded guard trace between two signal lines so as to reduce the electromagnetic coupling between them. However, the forward crosstalk traveling back and forth on the guard trace results in a series of voltage peaks in the near and far ends on the victim line. An intuitive weakly coupling analysis based on wave tracing is presented to explain the generation mechanism of such ringing noise. Also, an approximate analytic formula for the magnitude of the largest ringing noise of the near- and far-end crosstalk waveforms is derived from a multi-mode analysis of the three-line structure and verified through HSPICE.

Based on the aforementioned physical mechanism, the ringing noise can be completely eliminated by equalizing the capacitive and inductive coupling coefficients between the signal line and the guard trace. Hence, this paper proposes to take advantage of the solder-mask superstrate on microstrip structures, commonly used in printed circuit board manufacturing, thereby reducing the difference in the propagation delay of the modes involved in multi-line coupled systems. A design solution space for minimum ringing noise is constructed as a function of the thickness and dielectric constant of the superstrate. This enhanced microstrip guard trace structure can reduce the near- and far-end crosstalk without causing ringing noise. There is no need of more than two shorting vias on the guard trace if the superstrate is designed within the solution space. Full-wave transient simulation results and TDR measurement have also been performed to validate the proposed design methodology.

\section{ACKNOWLEDGMENT}

The authors would like to thank Prof. G.-H. Shiue in Chung Yuan Christian University for constructive suggestions and H.-H. Cheng, C.-C. Wang, and C.-T. Chiu in the Advanced Semiconductor Engineering inc. for providing helpful information.

\section{REFERENCES}

[1] B. D. Jarvis, "The effects of interconnections on high-speed logic circuits," IEEE Trans. Electron. Comput., vol. 12, pp. 476-487, Oct 1963.

[2] A. Feller, H. R. Kaupp, and J. J. Digiacomo, "Crosstalk and reflections in high-speed digital systems," Fall Jt. Computer Conf., Las Vegas, Nevada, pp. 511-525, Nov. 1965.

[3] S. H. Hall and H. L. Heck, Advanced Signal Integrity for High-Speed Digital System Design, Hoboken, NJ: Wiley, 2009, ch. 4.

[4] S.-K. Lee, K. Lee, H.-J. Park, and J.-Y. Sim, "FEXT-eliminated stub-alternated microstrip line for multi-gigabit/second parallel links," Electron. Lett., vol. 44, pp. 272-273, Feb. 2008.

[5] T. R. Gazizov, "Far-end crosstalk reduction in double-layered dielectric interconnects," IEEE Trans. Electromagn. Compat., vol. 43, pp. 566-572, Nov. 2001.

[6] P. Muthana and H. Kroger, "Behavior of short pulses on tightly coupled microstrip lines and reduction of crosstalk by using overlying dielectric," IEEE Trans. Adv. Packag., vol. 30, pp. 511-520, Aug. 2007.

[7] J. Chilo and T. Arnaud, "Coupling effects in the time domain for an interconnecting bus in high-speed GaAs logic circuits," IEEE Trans. Electron Devices, vol. ED-32, pp. 347-352, Mar. 1984.

[8] H. You and M. Soma, "Crosstalk analysis of interconnection lines and packages in high-speed integrated circuits," IEEE Trans. Circuits Syst., vol. 37, pp. 1019-1026, Aug. 1990.

[9] D. N. Ladd and G. I. Costache, "SPICE simulation used to characterize the cross-talk reduction effect of additional tracks grounded with vias on printed circuit boards," IEEE Trans. Circuits Syst. II, vol. 39, pp. 342-347, June 1992.

[10] I. Novak, B. Eged, and L. Hatvani, "Measurement by vector-network analyzer and simulation of crosstalk reduction on printed circuit boards with additional center traces," in Proc. IEEE Instru. Meas. Technol., Irvine, California, pp. 269-274, May 1993.

[11] J. C. Coetzee and J. Joubert, "Full-wave characterization of the crosstalk reduction effect of an additional grounded track introduced between two printed circuit tracks," IEEE Trans. Circuits Syst. I, vol. 43, pp. 553-558, July 1996.

[12] A. Suntives, A. Khajooeizadeh, and R. Abhari, "Using via 
fences for crosstalk reduction in PCB circuits," in 2006 IEEE Int. Symp. Electromagn. Compat., pp. 34-37, 14-18, Portland, Oregon, Aug. 2006.

[13] P. Rodriguez-Cepeda, M. Ribó, F.-J. Pajares, J.-R. Regué, A.-M. Sánchez, and A. Pérez, "Multimodal analysis of guard traces," in 2007 IEEE Int. Symp. Electromagn. Compat., pp. 1-5, Honolulu, Hawaii, July 2007.

[14] K. Lee, H.-B. Lee, H.-K. Jung, J.-Y. Sim, and H.-J. Park, “A serpentine guard trace to reduce the far-end crosstalk voltage and the crosstalk induced timing jitter of parallel microstrip lines," IEEE Trans. Adv. Packag., vol. 31, pp. 809-817, Nov. 2008.

[15] Y.-S. Cheng, W.-D. Guo, G.-H. Shiue, H.-H. Cheng, C.-C. Wang, and R.-B. Wu, "Fewest vias design for microstrip guard trace by using overlying dielectric," IEEE $17^{\text {th }}$ Topical Meeting Elect. Perform. Electro. Packag., San Jose, California, pp. 321-324, 27-29 Oct. 2008.

[16] HSPICE Simulation and Analysis User Guide, Synopsys Inc. (www.synopsys.com).

[17] C. R. Paul, "Solution of the transmission-line equations under the weak-coupling assumption," IEEE Trans. Electromagn. Compat., vol. 44, pp. 413-423, Aug. 2002.

[18] B. Young, Digital Signal Integrity: Modeling and Simulations with Interconnects and Packages. Upper Saddle River, NJ: Prentice-Hall, 2001, pp. 360-368.

[19] D. Pavlidis and H. L. Hartnagel, "The design and performance of three-line microstrip couplers," IEEE Trans. Microwave Theory Tech., vol. MTT-24, pp. 631-640, Oct. 1976.

[20] V. K. Tripathi, "On the analysis of symmetrical three-line microstrip circuits," IEEE Trans. Microwave Theory Tech., vol. MTT-25, pp.726-7292, Sep. 1977.

[21] CST Microwave Studio, ver. 2006, Computer Simulation Technology (http://www.cst.com). 